\title{
Respuestas
}

Año 16

No. 2

Diciembre 2011

ISSN 0122-820X

\section{Concepciones de justicia analizadas desde la idea de igualdad propuesta en la teoria de justicia de John Rawls*}

\author{
Laura Patricia Plata Lozada** $\mid$ Jesús Ernesto Urbina Cárdenas***
}

Recibido:

Septiembre 23 de 2011

Aceptado:

Diciembre 12 de 2011

\section{Resumen}

El presente estudio pretende comprender las concepciones de justicia de estudiantes de una universidad, internos en un establecimiento penitenciario de alta y mediana seguridad, desde la idea de igualdad de John Rawls. La investigación se desarrolló desde un enfoque cualitativo y se fundamentó epistemológicamente en la hermenéutica. Para cumplir con los objetivos del estudio se realizó una observación y análisis en tres (03) estudiantes internos de diferentes edades, patios, carreras y delitos por el cual fueron condenados. Metodológicamente el proceso de construcción de categorías se llevó a cabo de dos formas que son: las categorías iniciales y las categorías emergentes mediante el proceso de codificación abierta, axial y selectiva. La información recogida fue analizada desde un enfoque hermenéutico y buscó principalmente comprender e interpretar las concepciones que sobre Justicia tienen los participantes desde las perspectivas de John Rawls. Se utilizaron como métodos de recolección, herramientas como: la entrevista semi-estructurada, la encuesta y la revisión teórica con el fin de recolectar, sistematizar y analizar la información.

Palabras clave: Concepciones, justicia, libertad, igualdad, recompensa, convivencia, subjetividad, conflicto.

\section{Abstract}

The present study intends to understand the students justice conceptions of a University, internal in a prison establishment of high and medium security, since the conception of justice of John Rawls. The investigation developed since a qualitative focus and was supported epistemologically in the hermeneutic. To comply with the objectives of the study an observation was carried out and analysis in three (03) internal students of different ages, patios, careers and crimes by which were condemned. Methodologically the categories construction process was carried out of two forms that are: the initial categories and the emerging categories. The information collected was analyzed since a focus Hermenéutico and sought chiefly to understand and to interpret the conceptions that on Justice have the participants since the perspectives of John Rawls. They were utilized like methods of harvesting, 
tools as: the interview semi-structured, the survey and the theoretical review in order to collecting, to systematize and to analyze the information.

Keywords: Conceptions, justice, liberty, equality, reward, contact, subjectivity, conflict.

\section{Introducción}

E n el marco de la teoría de justicia propuesta por John Rawls en 1971, se establece la igualdad como uno de los dos principios fundamentales a los que todos los seres humanos tienen derecho sin discriminación alguna de raza, sexo, ideas políticas o creencias religiosas. El trabajo que se presenta en este artículo expone resultados parciales del análisis de la categoría "igualdad" del proyecto de investigación titulado "Concepciones de justicia de estudiantes de una universidad internos en un establecimiento penitenciario de alta y mediana seguridad". La categoría igualdad se encuentra soportada teóricamente en la teoría de justicia de John Rawls y en los treinta artículos que componen la declaración universal de los derechos humanos.

El propósito de este estudio es comprender la naturaleza de las concepciones de justicia en los estudiantes de la Universidad Francisco de Paula Santander internos en el establecimiento penitenciario de alta y mediana seguridad de Girón- Santander. Esta investigación parte de asumir a la justicia como un fenómeno netamente social y de considerar que las concepciones que expresan los estudiantes de una u otra forma reflejan las diferentes realidades y situaciones a las que han estado expuestos tanto en su contexto interno como externo. La palabra justicia refleja en las percepciones de los estudiantes internos un sinnúmero de significados, por lo que no se puede afirmar que existen dos personas con concepciones de justicia totalmente iguales, teniendo en cuenta que estas ideas fueron construidas a través de las experiencias vividas por cada uno de ellos en el transcurso de su vida, y probablemente estas experiencias sean total o parcialmente diferentes.

A través de la historia las teorías de justicia han jugado un papel fundamental en el desarrollo de las sociedades y en el surgimiento de paradigmas que regulan el comportamiento humano. Algunos autores afirman que existen mayores problemas de injusticias y desigualdades sociales, económicas y culturales en los países subdesarrollados, que a su vez son los que poseen el mayor número de poblaciones en condiciones de pobreza extrema y miseria $^{1}$...

Aunque existen muchos teóricos en el mundo que han conceptualizado el tema de la justicia como es el caso de John Rawls, en Colombia existen pocos estudios que aborden el problema de la justicia desde el término de las personas detenidas o en condición de internos. Por otra parte, para el caso de la

${ }^{1}$ Según el Departamento Nacional de Estadística (DANE): "En el 2009, la pobreza por ingreso se redujo en 0,5 puntos porcentuales frente a 2008, al pasar de 46 a 45,5 por ciento de la población nacional; la pobreza extrema, entre tanto, disminuyó 1,4 puntos porcentuales para el mismo período de referencia, según el más reciente informe de la Misión para el Empalme de las Series Empleo, Pobreza y Desigualdad (MESEP). Recuperado el 26 de Enero de 2011 de http://www.dane.gov.co/daneweb_V09/index.php?option=com_content\&view=article\&id=744:presentan-cifras-sobrepobreza-en-colombia\&catid=1:latest-news Durante los últimos doce meses, diciembre 2009 - noviembre 2010, la tasa global de participación fue $65,7 \%$, la tasa de ocupación 57,5\% y la tasa de desempleo 12,5\%. Recuperado el 26 de Enero de 2011 de: http://www.dane.gov.co/files/ investigaciones/boletines/ech/ech/bol_ech_nov10.pdf. 
pedagogía previa revisión en Colombia y en Hispanoamérica aún no existen evidencias escritas sobre esta temática.

En Colombia se hace necesario que personas comprometidas con la justicia, la convivencia y la paz realicen investigaciones que aborden temas como que el que se propone en esta investigación, contribuyendo al fortalecimiento de las teorías de justicia ya existentes.

Para comprender las concepciones de justicia esimportante conocer, estudiar, analizar y categorizar los relatos, las experiencias, preferencias y necesidades reales de las personas que participan en esta investigación y de las diferentes teorías de justicia a través de la historia de la humanidad. Por todo lo anterior y por la experiencia como tutora de la Universidad Francisco de Paula Santander en el establecimiento penitenciario de alta y mediana seguridad de Girón-Santander por aproximadamente más de cinco (5) años de trabajar, compartiendo y viviendo experiencias con los estudiantes de la UFPS internos, nació la necesidad de plantear la siguiente pregunta de investigación: ¿Qué concepciones de justicia desde la idea de igualdad expresan los estudiantes de una universidad internos en un establecimiento penitenciario de alta y mediana seguridad?

\section{Horizonte teórico}

\section{Declaración Universal de los Derechos} Humanos. La tesis encuentra un importante sustento teórico en la declaración universal de los derechos humanos. Por tal razón fue necesario estudiar y analizar dicha declaración además de la concepción de justicia de Jhon Rawls entre otros autores; en el recorrido de los treinta (30) artículos de Rawls se promueve la justicia y la igualdad, pilares fundamentales del presente trabajo, que facilitan la comprensión de las concepciones de justicia de los estudiantes internos (García-Añon,
1994; Casal, 2000; Vita, 2003; Teitel,2003; Teimil-García, 2009).

La declaración oficial de los derechos humanos fue promulgada por medio de la resolución 217 A (III) de 1948, en la cual se recogen aquellos derechos que son considerados básicos para la defensa de todos los seres humanos sin discriminación alguna. Esta declaración es la unión de pactos y protocolos internacionales y constituyen la carta internacional de derechos humanos que obliga a los estados firmantes a cumplirlos e incluirlos en sus respectivas cartas magnas o constituciones, exigiendo a los gobiernos a trabajar para que tanto los individuos como las instituciones promuevan mediante la enseñanza y la educación estos derechos y libertades fundamentales del hombre, y promoviendo el desarrollo de las relaciones amistosas entre las naciones (Abuchaibe, 2008; Draibe, 1994; DNE, 2010; Cortes, 2010).

\section{La teoría de la justicia de John Rawls}

La concepción de la justicia (Alvarado, 2005; Arango, 2000; Caballero, 2006; Gargarella, 1994; González-Soler, 1983) parte del establecimiento de dos principios fundamentales: el primero justifica el hecho de que cada persona que participa de una práctica o se ve afectada por ella deber ser libre, y el segundo principio habla de las desigualdades que invaden el diario vivir de nuestro mundo. Estos dos principios son fundamentales para que las personas creen sus propias concepciones de justicia (Rawls, 2006).

En la teoría propuesta por este autor, conocida como teoría ideal, se expone la importancia de conocer y estudiar las preferencias y necesidades reales de las personas que componen la sociedad objeto, y una vez se conozcan estas demandas, se procede a tomar las decisiones de distribución justa y equitativa de los bienes y servicios para todos. Esta distribución debe partir del establecimiento imparcial de un sistema 
justo en las instituciones del estado, quien es el responsable de velar por la justicia social. Plantea además que las instituciones del estado deben representar la justicia, y esta debe ser contemplada e interpretada como una virtud, asegurando que estas instituciones pueden llegar a hacer todo lo ineficientes e inútiles ó retrogradas que se pueda pero nunca injustas.

En un planteamiento hecho por Rawls en el texto "justicia como equidad", afirma que es imposible encontrar una sociedad donde no existan injusticias o desigualdades pero lo realmente importante es que estas desigualdades no sean aceptadas, ni vividas, y que sean desterradas del todo de los pensamientos y de las actuaciones de las personas que componen esta sociedad, y de esta forma lograr un ideal social. "En un sociedad justa, las libertades de la igualdad de ciudadanía se toman como establecidas definitivamente; los derechos asegurados por la justicia no están sujetos a regateos políticos ni al cálculo de intereses sociales.... Siendo las primeras virtudes de la actividad humana, la verdad y la justicia no pueden estar sujetas a transacciones". (Rawls, 1971).

Para Rawls (2006), los principios que expresan la justicia corresponde a tres ideas: libertad, iguadad y recopensa.

Principios de justicia de John Rawls: Para Rawls el establecimiento de una sociedad justa y estable parte del principio de que sus ciudadanos se sientan, crezcan y vivan libres e iguales, sin importar los conflictos que se puedan presentar por el afán de satisfacer necesidades individuales, y sin interesar sus creencias éticas, morales, políticas, económicas y religiosas y de sus posiciones originales.

Primer principio: "Cada persona debe tener un derecho igual al esquema más extenso de libertades básicas iguales compatible con un esquema similar de libertades para otros". (Rawls, 2006:53).

Libertad: El concepto de libertad para Rawls se encuentra claramente definido en lo que él mismo denominó como el primer principio de justicia. Este primer principio es catalogado como un principio de libertades o de distribución equitativa de los más amplios esquemas de libertades básicas en igualdad de derechos y oportunidades para todos. Las libertades básicas de los ciudadanos son la libertad política (Votar y postularse a cargos de elección), libertad de expresión y de asociación, libertad de conciencia y libertad de pensamiento, libertad de la persona al mismo tiempo que el derecho de poseer propiedad personal, y libertad de no ser objeto de detención arbitraria.

Segundo principio: "Las desigualdades sociales y económicas deben de resolverse de modo tal que:

- Resulten en el mayor beneficio de los miembros menos aventajados de la sociedad (el principio de la diferencia).

- Los cargos y puestos deben de estar abiertos para todos bajo condiciones de igualdad de oportunidades (justa igualdad de oportunidades)". (Rawls, J. 2006:303)

Igualdad: El concepto de igualdad para Rawls se encuentra claramente definido en lo que él mismo denominó como el segundo principio de justicia.

Siguiendo a este autor, este segundo principio es catalogado como el principio de diferencia. El principio de la diferencia corresponde a la idea de la fraternidad porque incluye la necesidad de no querer mayores ventajas a menos que beneficien a los peor situados. Las desigualdades económicas y sociales habrán de ser conformadas, de modo tal que a la vez: a) se espere razonablemente que sean ventajosas para todos, b) se vinculen a empleos y cargos alcanzables para todos. Esto significa que no pueden intercambiarse las libertades aseguradas por el primer principio para obtener mayores ventajas económicas. Las desigualdades económicas deben apoyarse, por su parte, en el principio de la justa igualdad de oportunidades.

En este principio se introduce el concepto
Diciembre 2011 ISSN 0122-820X 
de eficacia sin olvidar la prioridad que tiene la justicia frente a la eficacia, aunque estos dos términos estén íntimamente ligados. El principio de eficacia o criterio de Pareto dice que el bienestar de un grupo está en su punto óptimo cuando es imposible que ninguno de sus integrantes mejore sin que al menos otro se vea perjudicado. Este principio de eficacia permite grandes desigualdades y por lo tanto distribuciones injustas como las que se darían, por ejemplo, en el caso de un sistema de servidumbre que no pudiera ser reformado para mejorar la condición de un siervo sin empeorar la condición de un terrateniente. El principio de la diferencia impediría estas desigualdades profundas al hacer trabajar toda desigualdad a favor de los menos favorecidos, optando por una distribución eficaz, a saber, aquella que no es posible reformar sin empeorar las expectativas de al menos uno; el peor situado. "Las expectativas más elevadas de quienes están mejor situadas son justas si y sólo si funcionan como parte de un esquema que mejora las expectativas de los miembros menos favorecidos. (Rawls, J. 1971: 80)

Según Rawls, las personas en la posición original optarían, una vez garantizadas las libertades básicas y la justa igualdad de oportunidades, por una distribución desigual de los otros bienes primarios como son la riqueza, la autoridad y el ingreso, si esta distribución desigual mejorara las expectativas de los menos favorecidos, es decir, les otorgara mayor bienestar que el que obtendrían con una distribución equitativa. "Un sistema social justo define el ámbito dentro del cual los individuos tienen que desarrollar sus objetivos, proporcionando un marco de derechos y oportunidades así como los medios de satisfacción dentro de los cuales estos fines pueden ser perseguidos equitativamente" (Rawls, 1997, 42).

Los principios de la justicia según este autor son básicamente dos: la igualdad en la repartición de derechos y deberes básicos, y la igualdad social y económica en la distribución de la riqueza y la autoridad, o su desigualdad sólo en aquellos casos compensadores para todos y todas, y en particular para los menos aventajados de la sociedad. En este sentido, la justicia y sus dos principios tienen que estar en el punto de partida del contrato social compartido o del acuerdo original: "son los principios que las personas libres y racionales interesadas en promover sus propios intereses aceptarían en una posición inicial de igualdad como definitorios de los términos fundamentales de su asociación" (Rawls, 1997, p. 24).

La igualdad de los seres humanos estará definida desde su naturaleza moral y racional como sujetos de libre elección, lo que abre la perspectiva del pluralismo razonable de la teoría Rawlsiana, en las diferentes formas como los sujetos desde sus propios intereses se vinculan al sistema social definido como justo. A pesar de que algunos estudios limitan los miembros de la sociedad - en calidad de iguales - a los adultos, dejando por fuera de la reflexión posible a los niños y las niñas, el estudio retomó sus conceptos poniéndolos en juego en la población infantil: "Justo define el ámbito dentro del cual los individuos tienen que desarrollar sus objetivos, proporcionando un marco de derechos y oportunidades así como los medios de satisfacción dentro de los cuales estos fines pueden ser perseguidos equitativamente" (Rawls, 1997, p. 42).

\section{Metodología}

La investigación se desarrolló desde un enfoque cualitativo (Ricoeur, 2006), fundamentado epistemológicamente en la hermenéutica (Cárcamo, 2005) y metodológicamente el proceso de construcción de categorías se llevó a cabo siguiendo el modelo de Strauss y Corbin (2002): codificación abierta, axial y selectiva.

La información recogida fue analizada desde la teoría fundamentada y se buscó principalmente comprender e interpretar las concepciones que sobre justicia tienen los participantes desde las perspectivas de John Rawls. Se utilizaron como métodos de recolección, herramientas como: la entrevista semi-estructurada, la encuesta y la revisión 
teórica con el fin de recolectar, sistematizar y analizar la información. Las categorías emergen a partir del relato de las experiencias de los participantes y entendiendo entonces a las concepciones como todo aquello que una persona o participante de una investigación conoce de algún tema en específico.

El análisis se hizo a través de categorías según las concepciones de John Rawls y experiencias relatadas por los participantes. Para el tema de la confiabilidad y la validez se desarrolló la triangulación a partir de las correlaciones entre los datos emitidos por los participantes, los referentes teóricos y lo que como investigador se observó y vivió en la realidad.

\section{Resultados y discusión}

Para la interpretación se agruparon los tres casos de estudio y las diez encuestas realizadas a los directivos y guardianes en dos casos integrados: estos casos contienen todos los testimonios tanto de estudiantes como de guardianes que luego fueron clasificados en las categorías de análisis. Los relatos de los participantes se identifican con un código en letras y números.

El Caso Integrado $\mathrm{N}^{\circ} 1$ lo componen los participantes objeto de estudio que son estudiantes de tecnología en administración comercial y financiera, licenciatura en informática y licenciatura en educación física. Esimportante resaltarque paralos participantes no era nada fácil hablar de temas tan sensibles y cercanos a sus experiencias de vida y su condición social, ya que desafortunadamente han sido actores fehacientes de la injusticia, violencia y del conflicto colombiano, también es necesario rescatar la fluidez, el compromiso, la profundidad y la honestidad en sus relatos contribuyendo en la elaboración de la tesis y en la comprensión de las concepciones de justicia.
El segundo caso integrado, está conformado por las respuestas de diez directivos y guardianes del establecimiento penitenciario de alta y mediana seguridad de Girón-EPAMS. Estas respuestas son más precisas y destacan con mayor énfasis la concepción de justicia en relación con los internos del penal. El análisis permite conocer una nueva concepción de justicia, pero esta vez desde la perspectiva de quienes son los encargados de custodiar y vigilar a los internos. El presente estudio no pretende comprender las concepciones de justicia de los guardianes, sino abrir el horizonte de las concepciones de justicia, y de las implicaciones que éstas puedan acarrear en la convivencia diaria en las instalaciones del establecimiento penitenciario.

Al interpretar los comentarios de los estudiantes sobre las concepciones de justicia se puede decir que estos testimonios emitidos por los estudiantes internos se encuentran altamente correlacionados con la teoría de justicia y los principios propuestos por Jhon Rawls en 1971.

\section{Caso integrado $\mathrm{N}^{\circ} 1$. Categorías iniciales: concepciones de justicia}

\section{CATEGORÍA: IGUALDAD}

Desde los inicios de la humanidad el pueblo clama a gritos igualdad de derechos, deberes y oportunidades para todos los seres humanos, sin importar su condición social, económica, racial, sexual, religión a la que pertenece o sus opiniones políticas. Al analizar los testimonios de los estudiantes se observa que su concepción de justicia concibe a la igualdad como un principio que expresa, emana y fomenta justicia, lo cual coincide en principio con varias de las tesis de Rawls, sustentadas en el marco teórico de este texto. ISSN 0122-820X 
- $1^{\circ}$ Subcategoría: deberes, derechos y oportunidades para todos:

Las desigualdades económicas, políticas y sociales y la insatisfacción de las necesidades básicas del ser humano, deben ser resueltas con igualdad de derechos, deberes, oportunidades y ventajas para todos y sin ningún tipo de discriminación, donde todos tengamos los mismos derechos y podamos acceder equitativamente a los mismos empleos. Las sociedades que no combaten efectivamente estas desigualdades tienden a desaparecer a futuro, ya que estas carencias generan un sinnúmero de problemas como la pobreza extrema, violencia, conflictos, etc. Si estas sociedades desean continuar en continuo crecimiento y desarrollo, el estado y sus instituciones deben empezar por distribuir equitativamente los recursos y los bienes, para que todos los individuos tengan igualdad de derechos, deberes y oportunidades para todos, además los individuos deben dejar a un lado sus intereses personales e individuales por la búsqueda de un bien común, primar el interés general por encima del particular: "Si, bastante porque, (...) como lo mencionaba (...) que todos tengamos lo mismo, para mi es justo que todos tengamos los mismos derechos, (...) los mismos valores, porque en este país hay clase media, alta y baja, y eso no es justo (...) porque se dice que aquí tenemos baja producción (...) cuando aproximadamente el ingreso per cápita es muy pobre, y resulta que aquí hay gente que tiene que sobrevivir con dos mil pesos en el día y hay gente que gana aquí 2, 3, 4 millones en un mes como hay otros que ganan 15, 20, entonces yo creo que no hay equidad, y la justicia va de la mano con la equidad" (E1:P27).

La igualdad de derechos, deberes y oportunidades incluyen también a quienes por circunstancias de la vida han cometido delitos y le han hecho daño a una sociedad. La igualdad es incluyente: "Sí, la justicia tiene que ver con la igualdad en todo los ámbitos. ¿Por qué?... Porque si hay justicia habrá igualdad de derechos y deberes tanto para los ciudadanos, como para los que cometen delitos" (E2:P23).
Los estudiantes contemplan la eliminación de las desigualdades como una oportunidad de solucionar los conflictos: "La justicia para mi es algo que se lleva a cabo frente a un problema, ya que en ella podemos encontrar la igualdad frente a derechos y deberes que se tengan en los problemas a solucionar" (E2:P9).

Los estudiantes consideran que una oportuna y eficiente intervención de los poderes del estado corrige desigualdades, disminuye la corrupción y hace cumplir la ley de igual manera para todos incluyendo al mismo estado y sus instituciones: “(...) como lo nombré anteriormente, con los falsos positivos que se están viendo hoy en día, (...) creo que con lo que están haciendo de coger los paramilitares, como que ya se ha ido cayendo un poco esa injusticia que había que todo el mundo hacía las cosas y quedaban tapadas. El mismo gobierno, gente del estado esta acá y esta hoy en día en la cárcel porque están participando en actos de corrupción, entonces como que se está viendo un poco de justicia porque esa gente que hacían sus delitos y como eran del estado nadie los podía tocar, pero hoy en día están empezando a pagar... ya como que vemos un poco de justicia en la justicia..." (E1:P21).

- $2^{\circ}$ Subcategoría: distribución equitativa:

Los estudiantes consideran que no se puede permitir que mientras unos se sacrifican y pasan dificultades, y que a su vez estos sacrificios son insuficientes, otros disfruten con ventajas estos sacrificios. El estado debe velar que exista una correcta y equitativa distribución de la riqueza, la autoridad, los ingresos y los empleos para todos los habitantes del país, sin discriminación alguna, todas las regiones deben ser tratadas por igual en todos los aspectos. La distribución inequitativa de los recursos para algunos departamentos de Colombia viola el segundo principio de Rawls en donde se expresa que debe existir un mayor beneficio para los menos favorecidos, y no para los más favorecidos de la sociedad, ya que esto provoca insatisfacción de las necesidades 
básicas del ser humano y se agudiza el conflicto: "(...) también me parece injusto lo que hace el estado con algunos departamentos, ya que los tiene totalmente olvidados como es el Chocó, me parece injusto que siendo un país como tan unido, nuestro país que es del gobierno del pueblo (...) concluyente, donde se eligen (...) donde el pueblo elige a sus gobierno democrático, y aquél lo que hace es prometerles y no les cumplen, me parece algo injusto, la pobreza que hay (...) en algunos lados el hambre que se vive en la gente que vive en casitas de cartón y sabiendo que tienen derecho por la Constitución a que se les brinde educación (...) un techo. Me parece totalmente injusto por parte del gobierno" (E1:P19).

El rico quiere ser cada vez más rico y el pobre tiene que resignarse a ser cada vez más pobre, la igualdad consiste en lograr que las expectativas de quienes están mejor situados, actúen para mejorar las expectativas de los menos favorecidos. Es necesario que los individuos conciban que sea necesario para que una sociedad se desarrolle y no desaparezca con el transcurrir del tiempo, hacer a un lado sus intereses particulares e individuales y dar paso a los intereses generales que beneficien a todos, y no solamente al más poderoso o al más rico: "Todos los seres humanos nacemos libres e igual condiciones y es la misma sociedad la que impone los malos hábitos y a través de ellos nace la gran problemática actual: el rico quiere ser más rico, pero este grupo es muy pequeño. El pobre es cada día más pobre, pero este grupo es grande $y$ en el medio de ellos hay las oportunidades, y cuando de oportunidades se trata entra toda la injusticia" (E3:P35).

Los estudiantes expresan que la distribución equitativa también incluye el establecimiento de condenas equitativas al delito. Tienen menores y mejores condenas quienes han cometido delitos atroces a la humanidad, terrorismo, narcotráfico, secuestros como los paramilitares, y sin importar las víctimas, a otras personas: "Injusticia, por ejemplo en el caso de los paramilitares, como es posible que (...) hombres que hayan cometido delitos atroces, delitos de lesa humanidad, terrorismo, narcotráfico, secuestro como se llamen, vayan a cumplir condenas menores de ocho años, eso es algo injusto frente a Colombia y una burla para la sociedad Colombiana víctimas de la violencia por los grupos al márgenes de la ley" (E2:P19).

El estado debe velar para que se establezcan condenas equitativas a los delitos sin discriminación alguna o interés prticular, para que estos no queden en la impunidad y se generen nuevos conflictos: "Si bastantes, (...) con lo mismo que se ve hoy en día de los paramilitares, me parece injusto lo que está aplicando hoy en día el gobierno, que es una pena alternativa. Cómo es posible que una persona que haya cometido 500 hasta 1000 homicidios, que ellos mismos lo dicen... les dan una pena hoy en día de 8 años. Me parece algo injusto que una persona que tal vez por error cometió el delito trata de demostrar su inocencia con miles de formas y no le creen. Entonces aquí es más fácil que una persona que dice "yo maté 5 o 10... los tengo por allí enterrados, ajá... gracias por colaborar con la justicia", entonces le hacen una rebaja del 50\%, pero aquel que viene luchando toda una vida diciendo señores mi pelea es por un país más justo, en el tribunal no le creen. Me parece algo injusto, en esa parte no estoy de acuerdo con la justicia lo que están haciendo hoy en día, (...) por ejemplo el acceso carnal violento, si yo violé a la niña, ah! muchas gracias por su colaboración le damos una rebaja del 50\%, me parece que eso no es justicia, eso es lo más injusto que puede haber en la justicia colombiana" (E1:P23).

\section{Conclusiones}

Se puede concluir, al finalizar el proceso de interpretación de los comentarios de los estudiantes, que las concepciones de justicia emitidas por los estudiantes internos se encuentran correlacionadas con los principios de justicia establecidos en la teoría de Jhon Rawls en 1971 y la declaración universal de los derechos humanos de 1948.

Para los estudiantes la igualdad también es justicia: la igualdad de derechos, deberes y oportunidades sin discriminación alguna de 
sexo, raza, religión, opinión política, condición social o económica. Es justicia que todos los seres humanos tengan iguales derechos de acceder equitativamente a los mismos empleos y de satisfacer las necesidades básicas como el trabajo, vivienda, salud, educación, alimentación, recreación etc. Existe justicia cuando los más favorecidos trabajan en pro de los más necesitados, y que sean distribuidas equitativamente las riquezas para todos por igual, donde para todos exista la posibilidad de poseer propiedades económicas y donde se pueda acceder a la propiedad intelectual y cultural. Para los estudiantes también es justicia cuando se emiten condenas equitativas al delito y cuando el estado y sus instituciones intervienen para disminuir las desigualdades sociales y la corrupción.

Para el personal de guardia y directivos justicia es tratar a todos por igual, sin importar la condición social, económica, política, religiosa o sexual. Para los guardianes y el directivo, quien es también perteneciente a la guardia, las concepciones de justicia de cada individuo se forman a partir de las experiencias vividas a lo largo de la vida de cada uno de ellos.

Los guardianes al igual que los internos conciben la libertad, la igualdad y la recompensa como justicia, y la califican como una virtud que facilita la solución de un sinnúmero de conflictos en los cuales se ven inmersos todos los seres humanos.

\section{Referencias}

\section{bibliográficas}

Abuchaibe-Olano, Roberto Nicolás. 2008. Análisis comparativo del proceso de justicia y paz Colombiano: entre las políticas de impunidad y las demandas de justicia: Universidad de los Andes, Bogotá - Colombia.

Alvarado, Sara Victoria; Héctor Fabio Ospina, María Teresa Luna. 2005. Concepciones de justicia en niños y niñas que habitan contextos urbanos violentos: Universidad de Manizales - Colombia.

Arango, Rodolfo. 2000. "John Rawls y los Derechos Constitucionales". Universidad Nacional de Colombia, Bogotá - Colombia. Recuperado el 27 de enero de 2011 de: http:// books.google.es/books? $\mathrm{hl}=\mathrm{es} \& \mathrm{lr}=\& \mathrm{id}=844 \mathrm{z}$ N343KSMC\&oi $=$ fnd\&pg $=$ PA157\&dq $=$ con cepciones + de + justicia\&ots $=\mathrm{tNNoecTnkw}$ $\&$ sig $=$ OwLAOCliPbe_oLQkb-

$\mathrm{lcRn}_{\mathrm{c}} \mathrm{W} \mathrm{w} \mathrm{w}_{\mathrm{w}} \mathrm{v}_{\mathrm{v}}=$ onepage $\& \mathrm{q}=$ concepcion es\%20de\%20justicia\&f $=$ false

Asamblea General de las Naciones Unidas; (1948). Declaración Universal de los Derechos Humanos, Resolución 217 A (III) del 10 de diciembre de 1948". París - Francia.

Caballero, José Francisco. 2006. La Teoría de la Justicia de John Rawls: Voces y contextos Otoño, núm. II, año I.

Cárcamo, Héctor. 2005. Hermenéutica y Análisis Cualitativo Cinta de Moebio: Vol. Núm. 023. Universidad de Chile, Chile.

Casal, Paula. 2000. Ideas para una Teoría de Justicia universal con una intención cosmopolita. Keele University/Harvard University - Boston Estados Unidos.

Congreso de la República de Colombia. (2005). Ley 975 de 2005, Ley de Justicia y Paz. Colombia.

Cortes Rodas, Francisco. (2010). Una Crítica a las Teorías de Justicia Global: Al Realismo, A Rawls, Habermas y Pogge. Universidad de Antioquia - Colombia.

Departamento Nacional De Estadística. (2010). Recuperado el 26 de Enero de 2011 de: http://www.dane.gov.co/daneweb_V09/ index.php? option $=$ com_content\&view $=$ arti cle\&id = 744:presentan-cifras-sobre-pobrezaen-colombia\&catid =1:latest-news

Draibe, Sonia. 1994. Neoliberalismo y 
Concepciones de justicia analizadas desde la idea de igualdad propuesta en la teoria de

Políticas sociales: Reflexiones a partir de las Experiencias Latinoamericanas. Universidad de Campinas Unicamp - Brasil.

Gargarella, Roberto. (1999), en el estudio titulado: "Las teorías de la justicia después de Rawls. Un breve manual de filosofía política". Publicado en Ediciones Padios Iberoamérica, S.A. Barcelona- España.

García-Añon, José. 1994. La teoría de la justicia y los derechos morales en John Stuart Mill: Universidad Universitat de Valencia España.

Giordan, André; Gerard De Vecchi. 1999. Los orígenes del saber. De las concepciones personales a los conceptos científicos. Sevilla: Díada Editores.

González-Soler, María Dolores. 1983. Fundamentos, análisis y crítica de la teoría de la justicia de John Rawls, Universidad Complutense de Madrid - España.

Jaramillo Jefferson; Yesid Echeverri. 2006. Aproximación a las Concepciones de Justicia y Bien desde John Rawls y Milton Fisk. Pontificia Universidad Javeriana, Bogotá Colombia. Universidad de San Buenaventura, Cali - Colombia.

Martini, Florencia. 2009. La teoría de la Justicia entre el liberalismo igualitario y el marxismo contemporáneo - Teoría de la Justicia, Igualdad Jurídica, Libertad Individual, Liberalismo Igualitario, Teoría Democrática Deliberdora. Tierra socialista: Papeles sobre la Democracia, socialismo y ecología política, año 1. Universidad Nacional del Comahe Buenos Aires - Argentina.

Muñoz-Gaviria, Lina Paola. 2003. Hacia una observación de las concepciones de justicia en la toma de decisiones vistas en el caso de la conciliación: Tesis de Maestría en Ingeniería Industrial, Universidad de los Andes, Bogotá - Colombia.

Pérez de la Fuente, Oscar. 1993. "Escalas de Justicia y Emancipación: Inclusión,
Redistribución y Reconocimiento". Universidad Carlos III de Madrid - España.

Rawls, John. 2006. Teoría de la Justicia: revised edition, FCE, $6^{\underline{a}}$ reimpresión de la $2^{\underline{a}}$ edición.

Rawls, John. (2008). Microsoft@ Student 2009 [DVD]. Microsoft Corporation.

Rawls, John. Justicia como Equidad 1: Revista Española de Control Interno. W.D. LAMONT, the Principles of Moral Judgement (Oxford, 1946), cap. V.

Revista electrónica de investigación y evaluación educativa: Justicia: Concepciones filosóficas: Sofistas, Platón, Aristóteles, Santo Tomás de Aquino, Maquiavelo, Hobbes, Hume. Recuperado el 27 de Marzo de 2010 de http://www.ixquick.com/do/metasearch.pl

Ricoeur, Paul. Del Texto a la Acción. 2006. México, FC.

Teimil-García, Iván. 2009. Concepciones de la justicia en la filosofía política contemporánea, imparcialidad, universalismo y diferencia: Universidad de Oviedo - España.

Teitel, Ruti. 2003. Genealogía de la Justicia Transicional. Publicado en Harvard Human Rights Journal, Vol. 16. Cambridge, MA.

Vita, Álvaro. 2003. La Teoría de Rawls de la Justicia Internacional. En publicación: filosofía contemporánea. Controversias sobre civilización, imperio y ciudadanía. Atilio A. Borón. CLACSO, Consejo Latinoamericano de Ciencias Sociales, Ciudad Autónoma de Buenos Aires - Argentina.

Diciembre 2011
ISSN $0122-820 \mathrm{X}$ 